East African Medical Journal Vol. 86 (Supplement) December 2009

RADIATION SYNOVECTOMY: TREATMENT OPTION FOR HAEMOPHILIAC PATIENTS WITH CHRONIC HAEMARTHROSIS: A REVIEW

G. W. Kitonyi, MBChB, (UoN), FRCPath,(UK), Senior Lecturer, Department of Human Pathology, Haematology and Blood Transfusion Unit and J. M. Kitonyi, MBChB, (UoN) MMed (Radiol) (UoN), Cert. HCE, (Toronto), MSc (Nuclear Med), (London), Associate Professor, Department of Diagnostic Imaging and Radiation Medicine, College of Health Sciences, University of Nairobi, P. O. Box 19676 -00202, Nairobi, Kenya

Requesr for reprints to: Dr. G. W. Kitonyi, P.O. Box 21494 - 00505, Nairobi, Kenya

\title{
RADIATION SYNOVECTOMY: TREATMENT OPTION FOR HAEMOPHILIA PATIENTS WITH CHRONIC HAEMARTHROSIS: A REVIEW
}

\author{
G. W. KITONYI and J. M. KITONYI
}

\begin{abstract}
Background: Ablation of the synovium with radiopharmaceuticals, referred to as radiation synovectomy, (RS), has emerged as a simple affordable and safe procedure that is highly effective in preventing chronic disabling end stage arthritis in haemophilia patients.

Objective: To provide a review of the principles and role of radiation synovectomy, (RS), in the management of haemophiliac patients with chronic haemarthrosis, and to consider the possibility of this treatment option in Kenyan patients with haemophilia.

Data sources: A literature search through the internet using Boolean commands, PubMed interface to MEDLINE, Evidence, the Cochrane library. Papers from reputable haematology and radiation medicine journals, as well as conference presentations of the World Federation of Hemophilia were also included.

Data selection: The searches for papers, abstracts and reviews were limited to English language, haemophilia, haemarthrosis, synovectomy, RS, radiopharmaceuticals for RS and safety of RS.

Data extraction: All abstracts, and most of the papers were reviewed. Only those abstracts, papers and conference materials from reputable sources were used for this paper.

Data synthesis: All available papers and abstracts were reviewed for the most up to date information. The indications, requirements, procedure and safety aspects of RS were examined. The merits of alternative forms of synovectomy were considered. In the light of this information, the feasibility of RS as a treatment option for haemophilia patients in Kenya was considered.

Conclusion: Radiation synovectomy has been carried out on thousands of haemophilia patients with chronic haemathrosis over the last 20 years. Experience accumulated in numerous centres in America, Europe, Asia, North and South Africa indicates that majority of haemophilia patients undergoing RS are spared life long crippling arthritis and deformity. The principle safety concern of the potential for late radiation- related malignancy has been mitigated by appropriate choice of radiopharmaceuticals, and carefully executed procedure. The effectiveness, simplicity and affordability of RS make it particularly suitable for treatment of haemophilia patients in developing countries like Kenya.
\end{abstract}

\section{INTRODUCTION}

Haemarthrosis is the most painful and the most physically and psychologically debilitating manifestation of severe haemophilia (1). Frequently affected joints are the knees, anklejoints, elbows and the shoulders in that order. Bleeding usually commences in early childhood (2). Repeated haemarthrosis eventually leads to hypertrophy of the synovium and chronic synovitis (3). The inflammed hypertrophied and highly vascular synovium is prone to frequent haemorrhages, leading to chronic haemarthrosis. A chronic self-perpetuating cycle of haemarthrosissynovitis-haemarthroses is established in the target joint (4). Normally the breakdown products of blood, including iron, are removed by the synovium. In chronic synovitis the synovium is overwhelmed and the breakdown products lead to destruction of the articular cartilage and irreversible degeration and destruction of the bone $(5,6)$. 
The ideal way to prevent chronic synovitis is by prophylactic administration of clotting factors to prevent bleeds, beginning in infancy, after the first bleed and continuing indefinitely (7). This prophylactic treatment approach iscommon in developed countries. Indeed chronic synovitis is no longer a problem in developed countries (8). Unfortunately prophylactic factor concentrate therapy, and on- demand therapy are highly expensive, and therefore are not affordable by patients in resource poor setups. Many haemophilia patients in these countries eventually develop chronic synovitis followed by end stage disabling arthritis. Once chronic synovitis has set in, the only way of preventing irreversible joint damage is by removal of the synovium $(9,10)$. Synovectomy is therefore key to prevention against lifelong disabling arthritis in haemophilia patients who have chronic synovitis. Synovectomy can be accomplished surgically, or by non-surgical procedures (11-13).

Radiation synovectomy is not a first line treatment for haemarthroses in centres where factor concentrates are available and affordable, but rather an alternative or supplementary treatment. In the developing world where factor concentrates are prohibitively expensive, RS has been recommended to prevent joint damage. Imaging of the joint with MRI or conventional radiography or ultrasonography must be undertaken before RS, to establish that the joint is not already damaged since patients with damaged joints do not benefit from RS $(11,12)$.

Surgical synovectomy: This is undertaken through an open incision, (open synovectomy), or through arthroscopy, under general anaesthesia $(14,15)$. Open synovectomy requires a major hospital stay and lengthy rehabilitation and can cause a loss of joint motion. Large amounts of clotting factor concentrates are required in haemophiliacs, making the operation extremely expensive. Arthroscopic synovectomy requires shorter hospitalisation, and may preserve motion but still requires massiveamounts of prophylactic factor therapy. The success rate of surgical synovectomy in controlling recurrent bleeds is $80 \%$ but the cost is high $(11,12)$. The cost of surgical synovectomy in a haemophilia patient in the USA has been estimated at US $\$ 61,000$ (12).

Chemical synovectomy: Chemicals introduced into a joint cavity may cause sclerosis of the synovium. This approach has been used with schrosing agents like thiotepa, osmic acid, D-pencillaminase, oxytetracycline and rifampicin $(16,17)$. These chemicals are readily available and relatively inexpensive. Their usehowever is associated with limited clinical success and multiple injections are necessary for satisfactory results. Despite the use of local anaesthetics, injections of these drugs are very painful rendering them unpopular with patients (11).
Radiation synovectomy: Radiation synovectomy, radionuclide synovectomy, radio synovectomy, radiosynoviorthesis and radioactive synovectomy are all terms commonly used to describe synovial ablation induced by intra-articular injection of radioisotopes $(11,12,18)$. RS transforms richly vascularised synovium to sclerotic poorly vascularised tissuethat does notbleed easily. RShas been in use for treatment of different types of arthritis, including rheumatoid arthritis since theearly 1960's (19). Thousands of patients, including children, have undergone $\mathrm{RS}$ in many parts of the world to date (20-23).Countries where RS treatment of haemophilia has been reported include North and South America, Europe, Asia, the Far East, South Africa and someNorth African countries (12,23-27). The mid and longterm efficacy of RS is $75-80 \%$ as measured by decrease, (or disappearance), of the number of haem-arthroses (28, 29). The aim of RS therapy is to prevent arthropathy associated with recurrent haemarthrosis and synovitis $(11,12)$. Ideally the RS haemophilia candidate is the patient who has recurrent chronic haemarthrosis, but whohas no, (or has minimum), radiological evidence of joint damage. Since RS does not reverse already existing damage, RS should be started early in the patient with chronic synovitis, (29). Imaging of the target joint is recommended before RS for grading of arthropathy (30). Magnetic Resonance Imaging (MRI) is more sensitive than conventional radiographic studies for joint assessment, butinthe absence of MRI, combination of ultrasonography and conventional radiography has proved to be satisfactory (31-33).

A successful RS programme requires a well coordinated multidisplinary team of medical specialists (11), including musculoskeletal physicians, radiologists, nuclear physicians, haematologists, physical therapists and appropriately trained nurses (12). The radiologist is key in assessing the suitability of the target joint for RS. Injection of the radiopharceutical into the joint is usually carried out by the musculoskeletal or nuclear medicine physician. Physiotherapy and nursing care is undertaken by physiotherapists and nurses familiar with the care of haemophiliacs. Haematologists coordinates the process and ensures that the coagulation factor concentrateis administered before the procedure and the desirable level of the coagulation factor is achieved to avoid bleeding during the procedure. The programme should ideally be located in a designated haemophilia treatment centre where there are experts in haemophilia management.

Choice of radioisotopes: Different radiopharmaceuticals including Gold, ( $\left.{ }^{198} \mathrm{Au}\right),(22),{ }^{32} \mathrm{P}$ chromate phosphate, (34), Yttrium ${ }^{90},(23)$, and Dysprosium ${ }^{66},(25)$, have been used for RS. The preferred isotope is one with pure beta-emissions, a shallow depth of penetration, (to avoid radiation of surrounding tissue), and a moderate half life, to allow for gradual energy 
deposition avoiding immediate inflammatory reactions associated with rapidly decaying isotopes (35). Yttrium $\left.{ }^{90},{ }^{90} \mathrm{Y}\right),{ }^{32} \mathrm{p}$ chromic phosphate, ${ }^{32} \mathrm{p}$ ), and Dysporosium $^{66}$ are pure beta- emitters commonly used for RS. ${ }^{32} \mathrm{P}$ is the agent of choice in NorthAmerica, while ${ }^{90} Y$ is more widely used in Asia; South America, Europe and South Africa (11).Leakage of radioisotope outside the joint may cause systemic irradiation. To avoid leakage the radioisotopes are normally bound to a colloid carrier (36).

Injection procedure: Most centres perform RS as an out-patient procedure following a dose of clotting factor infusion to a level of around 50\%, $(11,12,24)$. Haemarthrosis is evacuated by arthrocentesis before RS following local anaesthetic injection into the joint cavity.Intra-articularinjection of radioisotopes has been described as virtually painless. Rodriguez- Merchan (29) has recommended an overnight stay for the few patients who may haveinhibitors. Echo-graphicguided injection has also been recommended, where facilities allow, but is not mandatory (12).Addition of steroid injections into the joint following the radioisotope injection, to reduce post - irradiation inflammatory response, is controversial (23). The first injection may not be sufficiently effective in $20-25 \%$ of patients, and a small proportion of patients may require up to three injections at 3-6 monthly intervals $(11,12)$.

Safety issues: The major safety concern of RS is the potential for radiation associated malignancy. Chromosomal changes after RS have been studied extensively (37). Nonspecific chromosomal changes have been described after RS in a small proportion of patients following RS with beta emitters but these changes disappear within a year of the procedure (38). No structural pre-malignant changes have been described in these patients. There was concern when acute lymphocytic leukaemia (ALL) was described in two young haemopihiliacs a few years after they underwent RS (39). However when adjusted for the expected incidence of ALL in the population, it was not clear whether this represented coincidental events that occur in accordance with population statistics. Along-term follow up of over 5000 patients of patients treated with RS did not reveal any radiation induced malignancy (28).

Other safety concerns are related to local radiation effects on the articular cartilage or growth plate disturbance in children. The articular cartilage has been shown to be very resistant to radiation and no damage has been reported in hundreds of children who have undergone RS over the last 20 years (12). Occasional radiation associated burns along the needletract have been reported but these have healed within six weeks (23). Nonetheless strict adherence to recommended procedure, and appropriate skill for the injection of the radioisotope, is paramount to avoid these side effects (10).

Costs:The cost of RS of a singlejoint has been estimated at US $\$ 150$ in Spain, andUS $\$ 3000$ in the United States of America (11). The weight of evidence indicates that RS is an affordable, minimally invasive procedure that is safe, and which greatly improves quality of life, by preventing incapacitating crippling end arthritis that inevitably follows chronic synovitis. It is currently recommended that developing countries should adopt RS for a healthier future generation of haemophilia patients.

\section{CONCLUSION}

In Kenya there are many patients afflicted by haemophilia and allied bleeding disorders (40). Many have severe haemophilia or Christmas disease and are frequently admitted for management of haemarthrosis. Clotting factor concentrates are unaffordable to the majority of patients in public health facilities for prophylactic treatment or on demand treatment of bleeds (41). Although small quantities of factor replacement concentrates are availed by donors from time to time in the public health facilities, the supplies are too few and erratic. The main treatment choices available for the management of these patients are cryoprecipitates or fresh frozen plasma, (FFP), often in combination with antifibrinolytic drugs. These products donot contain sufficient coangulation factors for adequate and effective treatment of haemarthrosis. Many of the patients thereforeend up with incomplete resolution of haemarthrosis and eventually develop chronic synovitis and subsequent end stage haemophilic arthropathy. For the significant number of patients who currently have chronic synovitis, there is an urgent need to consider the treatment option of RS to spare them from disabling end stage arthritis. Since the procedure of RS requires very little factor concentrates, some of the donated concentrates could be used to cover these procedures.

Kenya is well endowed with the necessary cadres of professional specialists for an RS programme. Most are familiar with the care of haemophilia care and only minimal further training would be required to start an RS programme in Kenya. RS would also reduce the risk of transmission of blood borne transmissible infections associated with repeated blood and blood products transfusion, in haemophilic patients. 


\section{REFERENCES}

1. Negrier, C., Goudemand, J. and Lambert T., Haemophilic arthropathy from A to Z. Hemophilia. 2008; 14 (Suppl. 4): 1-2.

2. Greer, P.J., Foerster J., Lukens, N. J., et al. Wintrobe's Clinical Haematology. $12^{\text {th }}$ Ed. Philadelphia, USA Lippincott Williams and Wilkins, 2009.

3. Lafeber, F. J., Miossec, P. and Valentino, A. Pathophysiology of haemophilic arthropathy. Hemophilia. 2008; 14 (Suppl. 4): 3-9.

4. Rodriguez-Merchan, E.C. Pathogenesis, early diagnosis and prophylaxis for chronic hemophilic synovitis. Clin. Orthop. 1997; 343: 6-11.

5. Arnold, W.D. and Hilgartiner, M. W. Hemophilic arthropathy. Current concepts of pathogenesis and management. J. Bone Joint Surg. Am. 1977; 59: 287305.

6. Roosendaal, G. and Lafeber F. P. Pathogenesis of haemophilic arthropathy. Haemophilia. 2006; 12 (Suppl. 3): 117- 121.

7. Raffin, C. L. and Manno, C. Modern management of haemophilic arthropathy. Br. J. Haematol. 2007; 136: 777-787.

8. van der Berg, H.M., Dunn, A., Fisher, K., et al. Prevention and treatment of musculoskeletal disease in the haemophilia population: role of prophylaxis and synovectomy. Hemophilia. 2006; 12 (Suppl. 3): 159-168.

9. Storti, E., Traldi, A., Tossatti, E., et al. Synovectomy, a new approach to haemophilic arthropathy. Acta. Haematol. 1969; 41:193 -205.

10. LLinas, A. Therole of synovectomy in themanagement of a target joint. Haemophilia. 2008; 14 (Suppl. 3): 177180.

11. Rodriguez- Merchan, E. C. Articular bleeding (Haemarthrosis), in hemophilia. An orthorpedist's point of view. Monograph series: Treatment of hemophilia 23 www.wfh.org. World Federation of Hemophilia, 2008. Last accessed in December 2009.

12. Silva, M. and Llinas, A. Chronic hemophilic synovitis Monograph series: The role of radiosynovectomy. Treatment of hemophilia, 33, www.wfh.org World Federation of Hemophilia ,2004. Last accessed in December 2009.

13. Kitchens, S.C., Alving, B. M. and Kessler C.M. Consultative Hemostasis and Thrombosis. 2nd Ed. Philadelphia, USA. Saunders Elservier, 2007.

14. Triantafyllou, S.J., Hanks, G.A., Handal, J.A., et al. Open and arthroscopic synovectomy in hemophilic arthropathy of the knee. Clin. Orthorp. 1992; 283: 196-204.

15. Luck, J.V. and Kasper, C.K. Surgical management of advanced hemophilic arthropathy. An overview of 20 years experience. Clin. Orthop. 1989; 242: 60-82.

16. Caviglia, H.A., Fernadez-Palazzi, F., Maffei, E., et al. Chemical synoviorthesis for hemohilic synovitis. Clin. Orthop.1997; 343: 30-36.
17. Heim, M. The treatment of intra-articular synovitis by the use of chemical and radioactive substances. Hemophilia. 2002; 8: 369-371.

18. Manco-Johnson, M. J., Nuss, R., Lear, J., et al. Radiosynoviothesis in children with hemophilia. J. Peditr. Hematol. Oncol. 2002: 24: 534-539.

19. Ansell, B.M., Crook, A. and Mallard, J. Evauation of intra-articular colloidal gold ${ }^{198} \mathrm{Au}$, in the treatment of persistent knee effusions. Ann. Rheum. Dis. 1963; 22: 435-439.

20. Ahlberg, A. and Pettersson, H. Synorviorthesis with radioactive gold in hemophiliacs. Clinical and radiological follow up. Acta. Orthop. Scand. 1979; 50: 513-517.

21. Fernandez-Pallazi, F., de Boch, N.B. and de Vargas, A F. Radioactive synovectomy in haemophilic haemarthrosis. Follow up of 50 cases. Scand. J. Haematol. Suppl. 1984; 40: 291-300.

22. Merchan, E., Magallon, M., Martin-Villar, J., et al. Long term follow up of haemophilic patients treated by 198 Au-radiation synovectomy. Int. Orthop. 1993; 17: 120- 124.

23. Kavakli K., Aydogdu S., Omay S.B., et al. Long-term evaluation of radioisotope Synovectomy with Yttrium 90 for chronic synovitis in Turkish haemophiliacs: Izmir Experience Haemophilia. 2006; 12: 28-35.

24. Marco- Johnson, M. J., Nuss, R., Lear, J., et al. Radiosynoviorsis in children with hemophilia. J. Pediatr. Hematol. Oncol. 2002; 24: 534-539.

25. Erken, E. H. Radiocolloids in the management of hemophilic arthropathy in children and adolescents. Clin. Orthop. 1991; 264: 129-134.

26. Klamboth, R., Gottstein, E., Stelling, E., et al. Radiosynoviorthesis in patients with haemophilia: Haemophilia carecentre, Berlin, Germany.Haemophilia. 2008; 14 (Suppl. 2): 83

27. Heim, M., Goshen, E. and Amit, Y.Synoviorthesis with radioactive Yttrium in haemophilia: Israel experience. Hemophilia. 2001; 7: 36-39.

28. Lee, P. The efficacy and safety of radio synovectomy. J. Rheumtol. 1982; 9: 165-168.

29. Rodriguez-Merchan,E.C.Radionuclidesynovectomy (radiosynoviothiorsis) in haemophilia: a very efficient and simple procedure. Sem. Thromb. Hemost. 2003; 29: 97-100.

30. Petterson, H., Ahlberg, A. and Nilsson, L.M. Aradiologic classification of hemophilic arthropathy. Clin. Orthop. Relat. Res. 1980; 149: 153-159.

31. Lundin, B., Pettersson, H. and Lujung, R. A new magnetic resonance imaging scoring method for assement of haemophilic arthropathy. Haemophilia. 2000; 6: 162-169.

32. Nuss, R., Kilcoyne, R.F., Geraghty, S., et al.MRI findings in haemophilic joints treated with radiosynoviorthesis with development of an MRI scale of joint damage. Haemophilia. 2000; 10: 383-389.

33. Seraphin-Krol, M.A. and Rockika-Milewska, R. Correlation between clinical, radiological and 
ultrasonographical image of knee joints in children with haemophilia. Haemophilia. 2001; 7: 286-292.

34. Silva, M., Luck, J.V. and Siegel, M.E. ${ }^{32} \mathrm{p}$ Chromic phosphate radio synovectomy for chronic haemophilic synovitis. Hemophilia. 2001: 7 Suppl 2: 40-49.

35. Rodriguez-Merchan, E.C.Synoviothesisinhemophilic patients: which is the best radioactive material to use? Haemophilia. 2005; 11: 191-192.

36. Siegel H. J., Luck J.V., Siegel M.E., et al. Hemarthrosis and synovitis associated with hemophilia: Clinical use of P-32 chromic sulphate phosphate synoviorthesis for treatment. Radiology. 1994; 190: 257 -261.

37. Fernadez- Pallazzi, F., Bosch, N.B. and Vargas, A. F. Chromosomal studies after radioactive synoviothesis for haemophilic haemarthroses. Int. Orthop. 1979; 3: $159-164$.
38. Falcon, D.V. and Fernandez-Palazzi, F. Cytogentic studies in patients with hemophilic hemarthroses treated by $198 \mathrm{Au}, 186 \mathrm{Rh}$, and 90y radioactive synoviothesis. J. Paediat. Orhop. 2000; 9: 52-54.

39. Dunn, A.L., Manco-Johnson, M., Busch, M., T.,et al. Leukaemia and p32 radionuclide synovectomy for hemophilic arthropathy. 2005; 3: J. Thromb. Haemost. 1541- 1542.

40. Kitonyi, G.W. and Kasili, E.G. Hereditary bleeding disorders in Kenya. E. Afr. Med. J. 1981; 58: 738-247.

41. Schnabell F. Status and Atlas of Hemophilia Worldwide. World Federation of Hemophilia.Quebec, Canada 1984. 